\section{TARMED bedeutet eine Gefahr für die Patienten}

R. R. Schmoker

Mitte November hatten die Fachgesellschaften erstmals Gelegenheit, die von Versichererseite für die Einführung vorgeschlagene Version des TARMED aus dem Internet herunterzuladen. Bei der Durchsicht des Tarifs gab es auf fachärztlicher Seite besorgte Gesichter. Viele Bestimmungen, Unzulänglichkeiten und Fehler im Tarif gefährden die Patientensicherheit oder verunmöglichen eine Anwendung des Tarifs. Dabei geht es nicht lediglich um noch fehlende Positionen, fehlerhaft geschriebenen Text oder falschen Kapiteln zugeordnete Begriffe usw. Bedenken wecken vielmehr von den Versicherern überall eingesetzte, die Patienten gefährdende Bestimmungen.

Eine adäquate Assistenz ist, unmittelbar nach dem Operateur, der wichtigste qualitätssichernde Faktor während einer Operation. Eine fehlende oder unzureichende Assistenz bildet anerkanntermassen häufig den auslösenden Faktor für intraoperative Zwischenfälle oder postoperative Komplikationen. Um die bei invasiver Tätigkeit bezüglich Qualitätssicherung zu Recht sehr hoch angesetzten Ansprüche zu erfüllen, muss es, je nach Einzelfall, abhängig vom Patientenrisiko, vom Schwierigkeitsgrad des Eingriffs, von gleichzeitig durchgeführten anderen Eingriffen und von der Institution, wo der beabsichtigte Eingriff durchgeführt wird, im Ermessen und in der Verantwortung des Operateurs liegen, ob und wie viele und ob ärztliche oder nichtärztliche Assistenzen indiziert sind.

\section{Assistenz am grünen Tisch geschätzt}

Im TARMED wurde die Assistenz von den Versicherern am grünen Tisch geschätzt und zu ganzen Zahlen auf- oder abgerundet, damit das Assistenzhonorar berechnet und dieses bereits fest in das Arzthonorar des Operateurs eingerechnet werden kann, unabhängig von der im individuellen Einzelfall effektiv benötigten und effektiv geleisteten Assistenz.

Korrespondenz:

Dr. med., Dr. med. dent. Roland R. Schmoker

Vorstandsmitglied FMS

Falkenhöhenweg 20

CH-3012 Bern
Darüber hinaus wurde die nichtärztliche Assistenz als Gratisleistung eingesetzt mit der Begründung, sie sei im Operationsteam bereits vorhanden. Dabei wurde vollständig vernachlässigt, dass es aus finanzieller Sicht für den Operateur oder das Spital von Bedeutung ist, ob bei einer Operation eine nichtärztliche Assistenz notwendig ist und mithilft oder nicht, da sie an anderer Stelle fehlt oder vorhanden ist. Gerade für den Belegarzt, der seine Assistenz selber mitbringt und selbst bezahlen muss, ist die Abrechnung der im individuellen Einzelfall geleisteten Assistenz, sowohl der ärztlichen wie der nichtärztlichen, von Wichtigkeit.

\section{Qualität wird leiden}

Bei der im TARMED von Versichererseite vorgeschlagenen pauschalen Einrechnung der Assistenz ist unter dem überbordenden Spardruck und angesichts des massiv gesenkten Tarifs die Versuchung gross, im Bereich der Assistenzen rigoros zu sparen und damit die Qualität der Behandlung zu gefährden. Der Operateur steht unter Druck, mit der im Tarif von Versichererseite eingesetzten Anzahl Assistenzen auskommen zu müssen oder eine nichtärztliche anstelle einer ärztlichen Assistenz zu akzeptieren. Sowohl er als auch das Spital geraten in Versuchung, mit weniger Assistenzen als pauschal im Tarif eingerechnet auszukommen oder unqualifizierte, billige Assistenzen einzusetzen. Diese Einsparung muss er nicht an die Versicherung oder den Patienten weiterleiten, da die Assistenz als Pauschale eingerechnet ist. Insgesamt gefährdet dies die Weiterbildung der Assistenten und damit der invasiv Tätigen allgemein.

Aus Gründen der Qualitätssicherung wird von fachärztlicher Seite eine Abgeltung je nach der im individuellen Einzelfall benötigten und auch wirklich geleisteten Assistenz über die ganze, oft aus mehreren Einzelschritten bestehende Gesamtoperation und auch über die Ein- und Ausleitzeit gefordert. Nur so kann die Qualität eines operativen Eingriffs gewährleistet bleiben.

\section{Qualitative Dignität (Zuordnung)}

Gefahr droht den Patienten auch von der eigenmächtig von Versichererseite vorgenommenen qualitativen Zuordnung der Operationen zu den einzelnen Fachdisziplinen. Die stümperhafte Art der Zuordnung hat unter den Fachspezialisten grosse Verärgerung ausgelöst. Teilweise fehlen pro Fachgesellschaft Dutzende von Operationen.

Nachdem die ärztliche Weiterbildung bis ins letzte Detail in einer allerseits anerkannten Weiterbildungsordnung exakt definiert und vorgeschrieben wird, fordern die Fachgesellschaften, dass sich die qualitative Dignität im TARMED nach dieser Weiterbildungsordnung zu richten hat und nicht nach den laienhaften Vorstellungen der Versicherer. 


\section{Automatische Sperren im Tarif}

Grossen Ärger ausgelöst haben die von Versichererseite teilweise unter offensichtlich fehlendem Verständnis für die Zusammenhänge eingebauten Sperren zwischen einer Vielzahl von Operationen. Die Überprüfung hat gezeigt, dass viele dieser Sperren eine optimale Kombination von Operationsschritten und damit eine auf den Einzelfall adäquat angepasste Behandlung verunmöglichen. Dies ist bei nahezu allen komplexen Eingriffen unabdingbar.

Ein typisches, von jedem Laien nachvollziehbares Beispiel sind die Patienten mit Lippen-Kiefer-Gaumen-Spalten (s. Kapitel 07.04 Spaltchirurgie). Bei jedem dieser Kinder sind oft zahlreiche Operationen notwendig. Bei solchen Eingriffen muss die optimale Kombination der einzelnen Operationsschritte genau geplant werden.

Die im TARMED von den Versicherern absolut dilettantisch eingesetzten Sperren verunmöglichen eine solche Planung, da nicht nur einzelne Eingriffe, sondern gerade die ganzen Kapitel, in denen solche in Frage kommenden Operationsschritte aufgezählt sind, gesperrt wurden (s. Kapitel 07.02, 07.03, 07.05, 07.07-07.12). Solche Sperren bei einem derart seltenen und schwierigen Patientengut, das nur von wenigen Operateuren betreut wird, stellen eine weitere, nicht begründbare Schikane dar. Radikaler lässt sich Arroganz und Ignoranz nicht mehr demonstrieren. Allein dieses Beispiel entspricht einem Skandal.

\section{Verantwortung im Operationstrakt}

Eine schwerwiegende Beeinträchtigung der Patientensicherheit wird dadurch entstehen, dass die Versicherer auch in der beabsichtigten Einführungsversion immer noch hartnäckig die Sockelzeit verweigern. Die Sockelzeit entspricht der sogenannten Ein-/Ausleitzeit im Operationstrakt vor und nach der eigentlichen Schnittnahtzeit. Ohne diese Sockelzeit kann ein Tarifmodell die Wirklichkeit im Operationssaal nicht abbilden. Bei kurzen Operationen beispielsweise werden dem Operateur für einen ganzen Tag Operationstätigkeit im Operationstrakt insgesamt lediglich 2-3 Stunden Schnittnaht- und Vor-/Nachbereitungszeit vergütet.
Damit sind der Operateur und die Assistenz für die übrige Zeit im Operationstrakt aus der Verantwortung gegenüber den Patienten entlassen. Solche Vorstellungen entsprechen einem laienhaften Denken. Verheerende Auswirkungen bis zur Verwechslung der Operationsseite usw. würden sich häufen. Haftpflichtrechtlich müsste jeweils abgeklärt werden, ob ein fehlerhaftes Vorgehen während der Schnittnahtbzw. Vor- und Nachbereitungszeit oder gerade während der für den Operateur und die Assistenz als «Freizeit» geltenden Ein- und Ausleitzeit vorgekommen ist.

Die hohen Ansprüche an die Qualitätssicherung im invasiven Bereich aller Fachgesellschaften verlangen, dass der Operateur und die Assistenz gemeinsam die volle Verantwortung für die chirurgischen Belange nicht nur während der Schnittnahtzeit und der Vor- und Nachbereitungszeit, sondern auch während der Ein-/Ausleitzeit zu tragen haben. Das Delegieren der ärztlichen Verantwortung, beispielsweise für die Lagerung, für die Bezeichnung und $\mathrm{Ab}$ grenzung des Operationsgebietes, für die Absprache der Anästhesieart mit dem Anästhesisten, für Zwischenfälle vor und nach der Operation usw. entspricht laienhaften Vorstellungen.

Selbst unter Einschränkung der Verantwortung des Operateurs auf die Schnittnaht- und Vor-/Nachbereitungszeit lässt sich die Tätigkeit des Operateurs nicht wie auf dem Fliessband aneinanderreihen. Daher resultiert bei Weglassung der Sockelzeit für kurze Eingriffe eine derart niedrige Abgeltung, dass die Patienten Mühe haben werden, für solche Eingriffe ein Spital, einen Operateur und eine Assistenz zu finden.

Auch der Ort und die Intensität der postoperativen Überwachung können einzig und allein vom Operateur und Anästhesisten, nie jedoch vom Versicherer festgelegt werden. Durch vorgegebene Sperren von Versichererseite, die eine adäquate Überwachung verhindern, sind die Patienten extrem gefährdet, z.B. Pos. 35.0110: Aufwachraum nur für stationäre Patienten. 